\title{
A Comparison of the Immediate Effects of Alternate Methods to Medication in Lowering the Blood Pressure in Prehypertensive Patients
}

\author{
Hyun-Ju Moon', Sung-Hak Cho² \\ 'Department of Physical Therapy, College of Health Science, Catholic University of Pusan, Busan; ${ }^{2}$ Department of Physical Therapy, College of Health \\ Science, Kaya University, Gimhae, Korea
}

Purpose: The purpose of this study was to compare the immediate effects of alternative treatment methods in lowering the blood pressure and pulse of prehypertensive patients.

Methods: A total of 48 prehypertensive patients were randomly divided into 4 groups of 12 patients and each group received a different intervention. The control group was made to rest, the heat pack (HP) group received heat pack treatment, the ultrasound (US) group received an ultrasound massage, and the myofascial release (MFR) group received myofascial release. The blood pressure and pulse rate were measured before and after intervention.

Results: Following intervention, the patients of the MFR group showed a decrease in systolic pressure, diastolic pressure, and pulse rate. Although blood pressure was similar in all groups, a significant decrease in pulse rate was seen in the MFR group compared to others.

Conclusion: The application of MFR was shown to be an effective method for lowering the blood pressure and pulse rate in prehypertensive patients.

Keywords: Prehypertensive, Myofascial release, Blood pressure

\section{INTRODUCTION}

Prehypertension, which affects $20-50 \%$ of the adult population worldwide, is defined as a resting systolic blood pressure of 120-139 $\mathrm{mmHg}$ and a diastolic pressure of 80-89 mmHg. ${ }^{1}$ When left untreated, prehypertension may progress to hypertension, which is an important risk factor for the development of various medical conditions, such as cardiovascular disease and stroke. ${ }^{2,3}$ Therefore, an important goal in prehypertensive patients is preventing its progression.

This goal is mainly achieved through lifestyle modification, as medications are not yet recommended. ${ }^{4}$ Many alternative methods, such as massage, exercise, thermotherapy, and myofascial release, have been used for managing blood pressure in prehypertensive patients. Massage has been known to lower blood pressure by increas-

Received May 25, 2016 Revised Jun 18, 2016

Accepted Jun 20, 2016

Corresponding author Sung-Hak Cho

E-mailwow1300@hanmail.net ing circulation and improving endothelial function through percutaneous mechanical stimulation of the blood vessels. ${ }^{5,6}$ Exercise helps patients maintain blood pressure homeostasis by improving regulatory ability. ${ }^{7.8}$ Thermotherapy also demonstrates a significant effect on lowering blood pressure ${ }^{9}$ by inducing vasodilation and promoting a response (baroreflex) of the autonomic nervous system. ${ }^{10}$ Finally, the release of fascia is used to relieve stress, alleviate pain, increase fluid mobilization and soft tissue mobility, and decrease heart rate, blood pressure, and anxiety, among others. ${ }^{11,12}$ In particular, myofascial release decreases the stiffness of the arteries by stimulating the parasympathetic nervous system, and activates endothelial function to control blood pressure. ${ }^{13}$ Various studies have showed that fascia release methods decreased a blood velocity of cerebral artery and a pain on tension type headache patients. ${ }^{14-16}$

Fascia forms a large network across the body, and a stimulation of

Copylight $@ 2016$ The Korea Society of Physical Therapy

This is an Open Access article distribute under the terms of the Creative Commons Attribution Non-commercial License (Http:// creativecommons.org/license/by-nc/4.O.) which permits unrestricted non-commercial use, distribution, and reproduction in any medium, provided the original work is properly cited. 
the fascia in one area can affect the entire body. ${ }^{17}$ The stimulation of the atlanto-occiput area is thought to be especially effective in controlling blood pressure, as the vagus nerve (a parasympathetic nerve) is adjacent to this area.

Although various alternative methods have been used for controlling blood pressure, there is no consensus as to which method is the most effective is not well understood. While there have been studies on these methods individually, no study directly compares the effects of each method during a fixed period of time.

Therefore, the purpose of this study was to determine which method is the most effective in controlling blood pressure in prehypertensive patients, and to suggest a treatment of choice for prehypertension.

\section{METHODS}

\section{Subjects}

In this study, 48 volunteers with prehypertension were divided into four groups of 12 patients through random sampling. Subjects were confined to patients 40-60 years old, with a resting systolic pressure of 120-139 mmHg and a diastolic pressure of 80-89 mmHg. In addition, patients were limited to potential stress groups with a Psychosocial Wellbeing Index-Short Form (PWI-SF) score of 4 and a Likert scale total score between 9-26 (less than 8: healthy, 9-26: latent stress, more than 27: high risk stress) to eliminate psychosocial stress variables. ${ }^{18}$ Patients with metabolic disorders and cardiovascular diseases were excluded from this study, as well as patients who have taken medications in the last 6 months. The study was approved by the Kaya University Research Ethics Committee (Kaya IRB-80).

\section{Experimental methods}

Prior to intervention, the systolic blood pressure, diastolic blood pressure and pulse rate of all patients were measured following a 10 -minute rest. The control group was made to rest in a supine position. The heat pack (HP) group had a heat pack applied to the neck and shoulders. The ultrasound (US) group received a pulsed ultrasound massage to the neck and shoulders, including the atlanto-occiput area, by compressing the subcutaneous layer in circular movements at a speed of $2 \mathrm{~cm} / \mathrm{sec}$ and strength of $3 \mathrm{~W} / \mathrm{cm}^{2}, 1 \mathrm{MHz}$. For patients in the myofascial release (MFR) group, the fascia of the atlanto-occiput area was stretched slowly in the cephalic direction by the examiner, using the 2nd and 5th fingers of both hands. ${ }^{19}$ Intervention was applied for 20 minutes in each group. Blood pressure was measured 10 minutes after intervention and a comparison between blood pressures prior to and following intervention was made. Blood pressure was measured at the right brachial artery (Digital Blood Pressure Monitor, UR-767, Japan) and pulse rate was measured at the right radial artery (pulse/min). All patient measurements were done in supine position. To control variables caused by the circadian rhythm (secreted metabolites, such as stress hormones), all measurements were carried out between 10:00 and 10:30 a.m.

\section{Statistical analysis}

A statistical analysis was carried out with SPSS 19.0 software (Chicago, IL, USA). The general characteristics of patients per group were presented using descriptive statistics. A paired t-test was used to compare the blood pressures and pulse rates among groups, before and after intervention. The differences in blood pressures and pulse rates were analyzed by one-way ANOVA, and post hoc analysis was performed using Bonferroni test. Significance level was set at $a=0.05$.

\section{RESULTS}

The general characteristics of the subjects are not significant difference as Table 1.

A significant decrease in Systolic blood pressure was observed in all groups except the control group $(\mathrm{p}<0.05)$ (Table 2). In particular, patients in the MFR group showed a significant decrease in systolic blood pressure, diastolic blood pressure and pulse rate $(\mathrm{p}<0.05)$ (Table 2). No difference in blood pressure was seen among the groups, but pulse rate significantly decreased in patients in the MFR group $(\mathrm{p}<0.05)($ Table 2$)$.

Table 1. Characteristics of the subjects $(\mathrm{N}=48)$

\begin{tabular}{lcccc}
\hline & Control group & HP group & US group & MFR group \\
\hline Age (year) & $55.2(1.0)$ & $54.9(1.9)$ & $53.3(2.5)$ & $54.2(2.7)$ \\
Sex (M/F) & $3 / 9$ & $2 / 8$ & $2 / 8$ & $3 / 9$ \\
Height (cm) & $157.6(3.9)$ & $157.3(4.9)$ & $157.1(4.1)$ & $158.3(3.3)$ \\
Weight (kg) & $69.6(5.9)$ & $69.6(6.6)$ & $68.4(3.1)$ & $69.2(3.8)$ \\
BMl $\left(\mathrm{kg} / \mathrm{m}^{2}\right)$ & $33.8(3.2)$ & $33.7(3.3)$ & $33.5(3.1)$ & $33.2(3.8)$ \\
\hline
\end{tabular}

Values are in mean (standard deviation).

HP: Heat pack, US: Ultrasound, MFR: Myofascial release. 
Table 2. Change in blood pressure and pulse rate per group after intervention

\begin{tabular}{|c|c|c|c|c|c|}
\hline & & Control group & HP group & US group & MFR group \\
\hline Systolic & Pre & $127.2 \pm 3.1$ & $126.9 \pm 2.1$ & $130.0 \pm 4.6$ & $131.5 \pm 6.7$ \\
\hline $\mathrm{BP}(\mathrm{mmHg})$ & Post & $123.4 \pm 5.2^{*}$ & $120.2 \pm 4.0^{*}$ & $124.7 \pm 5.8^{\star}$ & $123.8 \pm 7.5^{*}$ \\
\hline Diastolic & Pre & $5.0 \pm 3.8$ & $83.5 \pm 5.8$ & $81.5 \pm 7.5$ & $79.0 \pm 6.6$ \\
\hline $\mathrm{BP}(\mathrm{mmHg})$ & Post & $73.6 \pm 5.4$ & $77.8 \pm 8.6^{*}$ & $77.9 \pm 7.0$ & $74.2 \pm 6.2^{*}$ \\
\hline Pulse rate & Pre & $74.2 \pm 6.2$ & $68.4 \pm 8.1$ & $67.6 \pm 6.3$ & $69.3 \pm 3.8$ \\
\hline (pulse/min) & Post & $71.5 \pm 9.5^{a}$ & $68.9 \pm 6.1^{a}$ & $67.0 \pm 5.6^{a}$ & $63.1 \pm 3.8^{\mathrm{b}, *}$ \\
\hline
\end{tabular}

Values are in mean \pm standard deviation.

BP: Blood pressure.

${ }^{*} \mathrm{p}<0.05$, Post-hoc: $\mathrm{a}<\mathrm{b}, \mathrm{a}=$ control group, HP group, US group, $b=$ MFR group

\section{DISCUSSION}

The purpose of this study was to compare the immediate effects of alternative treatment methods in lowering the blood pressure and pulse rate of prehypertensive patients. The MFR group showed a significant decrease in systolic blood pressure, diastolic blood pressure and pulse rate. It is believed that the slow, gentle and gradual stimulation of the fascia and the Ruffini endings within caused the subsequent activation of the parasympathetic nervous system, which, in turn, caused a decrease in blood pressure and pulse rate. ${ }^{20}$ Kim et al. ${ }^{21}$ observed a decrease in cortisol (known as a stress hormone) levels following self-myofascial release and it was attributed to the suppression of the sympathetic nervous system through relaxation of the fascia. Therefore, it can be assumed that the relaxation of the fascia can cause activation of the parasympathetic nervous system and decrease blood pressure and pulse rate. In addition, Mense stated that myofascial release controlled the balance of the autonomic nervous system by stimulating mechanoreceptors 3 and $4,{ }^{22}$ and proposed that the method also induced stretching of the deep fascia surrounding the internal organs, causing a release of neurotransmitters which can affect the cardiovascular system. ${ }^{23}$

A significant decrease in systolic blood pressure was observed in all four groups, including the control group. While both the HP group and MFR group showed a significant decrease in diastolic blood pressure, only the MFR group showed a significant decrease in pulse rate. This is believed to be because heat packs only affect surface temperature, and cannot mechanically stimulate the deep fascia and its mechanoreceptors. In addition, although an ultrasound massage causes friction of the superficial blood vessels by stimulating the muscles, the application speed is much quicker than myofascial release and therefore, cannot stretch the fascia gradually.
This musculocutaneous stimulation may not be sufficient to affect deeper layers and control diastolic blood pressure and pulse rate. Systolic blood pressure reacts quickly to environmental changes, but the same cannot be said for diastolic blood pressure, which is more difficult to control even with medications. Nevertheless, myofascial release and heat packs were reported as effective alternatives to medication for decreasing diastolic blood pressure.

In contrast to blood pressure, a significant difference in pulse rate was observed among the groups, and the contrast between the MFR group and the HP group was particularly significant. Myofascial release was shown to be the only alternative method that could immediately decrease pulse rate. Similar to its effects on blood pressure, it is believed that myofascial release stretches the deep fascia and affects the internal organs and the cardiovascular system, inducing an immediate response.

Since medications are not generally used for patients with prehypertension, alternative methods, such as myofascial release, seem to be effective in controlling blood pressure and pulse rate, ultimately preventing the progression to hypertension.

This study was performed measure of blood pressure and pulse rate for a short term. So future longitudinal studies are necessary to identify differences and the long term effects of myofascial release for lowing blood pressure.

\section{REFERENCES}

1. Chobanian AV, Bakris GL, Black HR et al. The seventh report of the joint national committee on prevention, detection, evaluation, and treatment of high blood pressure: The JNC 7 report. JAMA. 2003;289(19): 2560-72.

2. Lloyd-Jones D, Adams R, Carnethon M et al. Heart disease and stroke statistics 2009 update: A report from the American heart association statistics committee and stroke statistics subcommittee. Circulation. 2009; 119(3):480-6.

3. Kim JH, Kim IH, Hwang BY. The hypertensive vascular tension-related signal transduction and the relationship of physical therapy. J Kor Phys Ther. 2008;20(4):35-42.

4. Burnier M. World health organization/international society of hypertension, guidelines: Blood pressure control and the implementation of guidelines in clinical practice: Can we fill the gap? J Hypertens. 2002; 20(7):1251-3.

5. Nelson NL. Massage therapy: Understanding the mechanisms of action on blood pressure. J Am Soc Hypertens. 2015;9(10):785-93.

6. Supa at I, Zakaria Z, Maskon O et al. Effects of Swedish massage therapy on blood pressure, heart rate, and inflammatory markers in hypertensive women. Evid Based Complement Alternat Med. 2013;2013:171852. 
7. Pescatello LS, Franklin BA, Fagard R et al. American college of sports medicine: American college of sports medicine position stand exercise and hypertension. Med Sci Sports Exerc. 2004;36(3):533-53.

8. Kim MH, Lee HJ. Effects of exercise intensity on hypertension prevalence in Korean men with high sodium intake. J Phys Ther Sci. 2015; 27(9):2753-7.

9. Sawatari H, Chishaki A, Miyazono M et al. Different physiological and subjective responses to the hyperthermia between young and older adults: Basic study for thermal therapy in cardiovascular diseases. J Gerontol A Biol Sci Med Sci. 2015;70(70):912-6.

10. Crandall CG, González-Alonso j. Cardiovascular function in the heatstressed human. Acta Physiol (Oxf). 2010;199(4):407-23.

11. Hernandez-Reif M, Field T, Krasnegor J et al. Lower back pain is reduced and range of motion increased after massage therapy. Int J Neurosci. 2001;106(3):131-45.

12. Lee JH, Kang DH, Kang JI. The effects of myofascial relaxation on blood flow velocity of the cranial artery and pain level in cervicogenic headache patients. J Kor Phys Ther. 2010;22(5):49-56.

13. Okamoto T, Masuhara M, Ikuta K. Acute effects of self-myofascial release using a foam roller on arterial function. J Strength Cond Res. 2014; 28(1):69-73

14. Park RJ, Kim JS, Lee IH et al. Effecs of electrotherapy on blood velocity of cranial artery in tension-type headache subjects. J Kor Phys Ther.
2000;12(3):349-59.

15. Han JM, Kim JS. The effects of ultrasound and myofascial release therapy on blood velocity of cranial artery intension-type headache subjects. J Kor Phys Ther.2003;15(1):196-209.

16. Lee MH, Han JM. Cerebral blood flow velocity measurement by TCD: the effects of interferential current. J Kor Phys Ther. 2005;17(2):126-47.

17. Schleip R, Jäger H, Klingler W. What is 'fascia'? a review of different nomenclatures. J Bodyw Mov Ther. 2012;16(4):496-502.

18. Kim KH, Kim JW, Kim SH. Influences of job stressors on psychosocial well-being, fatigue and sleep sufficiency among firefighters. Korean J Occup Environ Med. 2006;18(3):232-45.

19. Chaitow L. Cranial manipulation: Theory and practice. London: Churchill Livingstone. 1999:49-52.

20. Schleip R. Fascial plasticity-a new neurobiological explanation: Part 1. J Bodyw Mov Ther. 2003;7(2):11-9

21. Kim K, Park S, Goo BO et al. Effect of self-myofascial release on reduction of physical stress: A pilot study. J Phys Ther Sci. 2014;26(11):177981.

22. Mense S. Basic neurobiologic mechanisms of pain and analgesia. Am J Med. 1983;75(5):4-14.

23. Gladwell VF, Fletcher J, Patel $N$ et al. The influence of small fiber muscle mechanoreceptors on the cardiac vagus in humans. J Physiol. 2005; 567(2):713-21. 